\title{
Sub Hilbert spaces in a Bi-Disk
}

\author{
Niteesh Sahni ${ }^{1,2, *}$ \\ ${ }^{1}$ Dept. of Mathematics \\ ${ }^{2}$ Shiv Nadar University, Village Chithera, Tehsil Dadri, Dist. Gautam Budh Nagar, Uttar Pradesh (India) 203207
}

\section{Email address:}

niteesh.sahni@snu.edu.in (N. Sahni)

To cite this article:

Niteesh Sahni. Sub Hilbert Spaces in a Bi-Disk, Pure and Applied Mathematics Journals. Vol. 2, No. 2, 2013, pp.98-100. doi: $10.11648 /$ j.pamj.20130202.17

\begin{abstract}
Recently, Sahni and Singh [7] have solved an open problem posed by Yousefi and Hesameddini [12] regarding Hilbert spaces contained algebraically in the Hardy space $\mathrm{H}^{2}(\mathrm{~T})$. In fact the result obtained by Sahni and Singh is much more general than the open problem. In the present note we examine the validity of the main results of [7] and [12] in two variables.
\end{abstract}

Keywords: Hardy Space, Beurling Type Result, Isometry, Wold Type Decomposition

\section{Introduction}

In [12], Yousefi and Hesameddini have characterized the invariant subspaces of all Hilbert spaces $\mathrm{H}$ that are vector subspaces of the Hardy space $\mathrm{H}^{2}(\mathrm{~T})$, with $\langle., .\rangle_{H}$ (the inner product on $\mathrm{H}$ ) satisfying the following axioms:

A1.If there are four functions $f_{1}, f_{2,} g_{1}, g_{2} \in H$ such that $\left\langle f_{1}, g_{1}\right\rangle_{H}=\left\langle f_{2}, g_{2}\right\rangle_{H}$, then we have $\left\langle f_{1}, g_{1}\right\rangle_{H^{2}}=\left\langle f_{2}, g_{2}\right\rangle_{H^{2}}$.

A2. If $\phi$ is any inner function, then $\varphi f \in H$ and $\langle\varphi f, \varphi g\rangle_{H}=\langle f, g\rangle_{H}$ for all $\mathrm{f}, \mathrm{g} \in \mathrm{H}$.

The characterization of $\mathrm{H}$, however is left as the following open question in [12]: Is every Hilbert space $H$ satisfying axioms $\mathrm{A} 1$ and $\mathrm{A} 2$ of the form $\varphi H^{2}$, for some $\varphi \in H^{\infty}$, and does there exist a constant $\mathrm{k}$ such that $\langle f, g\rangle_{H}=k\langle f, g\rangle_{H^{2}}$ for every $f, g \in H$ ?

Sahni and Singh [7] have settled this open problem in the affirmative. They in fact prove a far more general version of the above problem. Below we record a special case of their main result which clearly generalized the open problem mentioned above:

Theorem 1.1 ([7], Corollary 3.2). Let $\mathrm{H}$ be a Hilbert space which is algebraically contained in $\mathrm{H} 2$ and invariant under multiplication by z. Further if the inner product on $\mathrm{H}$ satisfies the following conditions

$$
\begin{aligned}
& \text { i. }\langle z f, z g\rangle_{H}=\langle f, g\rangle_{H} \text { for all } \mathrm{f}, \mathrm{g} \in \mathrm{H} \text {; } \\
& \text { ii. } \quad \text { for any } f_{1}, f_{2,} g_{1}, g_{2} \in H \text { such that } \\
& \left\langle f_{1}, g_{1}\right\rangle_{H}=\left\langle f_{2}, g_{2}\right\rangle_{H} \text {, then we have }
\end{aligned}
$$

$$
\left\langle f_{1}, g_{1}\right\rangle_{H^{2}}=\left\langle f_{2}, g_{2}\right\rangle_{H^{2}}
$$

then there exists a unique inner function $b \in H^{\infty}$ and a constant k such that $H=b H^{2}$ and $\langle f, g\rangle_{H}=k\langle f, g\rangle_{H^{2}}$ for every f,g $\in \mathrm{H}$.

In [7], the authors use the above characterization of $\mathrm{H}$ to derive the following invariant subspace characterization:

Theorem 1.2 ([7], Theorem 3.3). Let $\mathrm{H}$ be a Hilbert space satisfying all the conditions of Theorem 1.1. If $\mathrm{M}$ is a closed subspace of $\mathrm{H}$ and invariant under multiplication by $\mathrm{z}$, then there exists a unique inner function $\varphi \in H^{\infty}$ such that $\mathrm{M}=\phi \mathrm{H}$.

It is further noted in [7] that the characterization obtained in [12] comes as a special case of Theorem 1.2. In the present paper, we obtain a two variable version of Theorem 1.1 for the Hardy space $\mathrm{H}_{2}\left(\mathrm{~T}_{2}\right)$ on the torus $\mathrm{T}_{2}$, and also examine the validity of Theorem 1.2 in two variables.

\section{Notations and Preliminary Results}

Let $\mathrm{T}$ denote the unit circle in the complex plane, and $\mathrm{T}^{2}$ stands for the cartesian product of $\mathrm{T}$, that is, $T^{2}=$ $\left\{\left(e^{i \theta_{1}}, e^{i \theta_{2}}\right): 0 \leq \theta_{1} \leq 2 \pi, 0 \leq \theta_{2} \leq 2 \pi\right\}$. We shall denote the coordinate functions $e^{i \theta_{1}}$ and $e^{i \theta_{2}}$ by $\mathrm{z}$ and $\mathrm{w}$ respectively. The Lebesgue space $\mathrm{L}^{2}\left(\mathrm{~T}^{2}\right)$ on the torus is a collection of complex valued functions fon the torus such that $\int|f|^{2} d m$ is finite, where $d m$ is the normalized Lebesgue measure on $\mathrm{T}^{2}$. It is well known that $\mathrm{L}^{2}\left(\mathrm{~T}^{2}\right)$ is a Hilbert space with respect to the inner product

$$
\langle f, g\rangle_{2}=\int_{T^{2}} f g d m,
$$

and the collection $\left\{z^{n} w^{m}: m, n \in \mathbb{Z}\right\}$ is an orthonormal basis for $\mathrm{L}^{2}\left(\mathrm{~T}^{2}\right)$. The Hardy space $\mathrm{H}^{2}\left(\mathrm{~T}^{2}\right)$ comprises of those $\mathrm{L}^{2}\left(\mathrm{~T}^{2}\right)$ functions $\mathrm{f}(\mathrm{z}, \mathrm{w})$ that have the Fourier expansion of the form $\sum_{m=0}^{\infty} \sum_{n=0}^{\infty} a_{m n} z^{m} w^{n}$, and hence the norm of $\mathrm{f}$ is given by $\|f\|_{2}=\left(\sum_{m=0}^{\infty} \sum_{n=0}^{\infty}\left|a_{m n}\right|^{2}\right)^{1 / 2}$. 
The space $\mathrm{H}^{2}\left(\mathrm{~T}^{2}\right)$ turns out to be a closed subspace of $\mathrm{L}^{2}\left(\mathrm{~T}^{2}\right)$ and hence a Hilbert space under the above norm. The class of essentially bounded functions in $\mathrm{H}^{2}\left(\mathrm{~T}^{2}\right)$ is denoted by $H^{\infty}$. Let $S_{1}$ and $S_{2}$ be operators on $\mathrm{H}^{2}\left(\mathrm{~T}^{2}\right)$ defined by $S_{1} f(z, w)=z f(z, w)$, and $S_{2} f(z, w)=w f(z, w)$ respectively.

A function $\phi \in \mathrm{H}^{2}\left(\mathrm{~T}^{2}\right)$ is called an inner function if $|\phi|=1$ a.e.. Equivalently, this means that $\langle\varphi f, \varphi g\rangle_{2}=\langle f, g\rangle_{2}$ for every $f, g \in \mathrm{H}^{2}\left(\mathrm{~T}^{2}\right)$.

Theorem 2.1 (Singh [11]). Let $M$ be a Hilbert space which is a vector subspace of $\mathrm{H}^{2}\left(\mathrm{~T}^{2}\right)$ such that $S_{1}(M) \subset M$, $S_{2}(M) \subset M$; and $S_{1}, S_{2}$ are doubly commuting isometries on $\mathrm{M}$. Then there exists $b \in H^{\infty}$ such that $\mathrm{M}=\mathrm{bH}^{2}\left(\mathrm{~T}^{2}\right)$, and $\|b f\|_{M}=\|f\|_{2}$ for all $\mathrm{f} \in \mathrm{H}^{2}\left(\mathrm{~T}^{2}\right)$.

The following lemma provides a necessary and sufficient condition for an $H^{\infty}$ function to be an inner function:

Lemma 2.2. A function $\varphi \in H^{\infty}$ is inner if and only if $\left\{z^{m} w^{n} \varphi: m . n=0,1,2, \ldots\right\}$ is an orthonormal set in $\mathrm{H}^{2}\left(\mathrm{~T}^{2}\right)$.

Proof. Suppose $\phi$ is inner. So $|\phi|=1$ a.e., and therefore

$\left\langle z^{m_{1}} w^{n_{1}} \varphi, z^{m_{2}} w^{n_{2}} \varphi\right\rangle_{2}=\left\langle z^{m_{1}} w^{n_{1}}, z^{m_{2}} w^{n_{2}}\right\rangle_{2}=$

$\left\{\right.$ if $\left(m_{1}, n_{1}\right)=\left(m_{2}, n_{2}\right)$

\{ 0 if $\left(m_{1}, n_{1}\right) \neq\left(m_{2}, n_{2}\right)$

Conversely, the orthonormality of the set $\left\{z^{m} w^{n} \varphi: m . n=0,1,2, \ldots\right\}$ yields the following equations: 0 ,

$\left\langle\varphi, \varphi z^{m} w^{n}\right\rangle_{2}=0=\overline{\left\langle\varphi, \varphi z^{m} w^{n}\right\rangle_{2}}$ for all $\mathrm{m}>0$ and $\left.\mathrm{n}\right\rangle$

$\left\langle\varphi z^{m}, \varphi w^{n}\right\rangle_{2}=0=\overline{\left\langle\varphi z^{m}, \varphi w^{n}\right\rangle_{2}}$ for all $\mathrm{m}>0$ and $\mathrm{n}>0$, $\left\langle\varphi, \varphi z^{m}\right\rangle_{2}=0=\overline{\left\langle\varphi, \varphi z^{m}\right\rangle_{2}}$ for all $\mathrm{m}>0$,

and $\left\langle\varphi, \varphi w^{n}\right\rangle_{2}=0=\overline{\left\langle\varphi, \varphi w^{n}\right\rangle_{2}}$ for all $\left.\mathrm{n}\right\rangle 0$.

From the above four equations we conclude that

$\int|\varphi|^{2} z^{m} w^{n} d m=0$ for all $(m, n) \neq(0,0)$.

Therefore $|\phi|^{2}$ is a constant, say c.

Since $1=\|\varphi\|_{2}=\int|\varphi|^{2} d m$, we have $\mathrm{c}=1$. This proves that $\phi$ is inner.

\section{Main Results}

Theorem 3.1. Let $H$ be a Hilbert space which is a vector subspace of $\mathrm{H}^{2}\left(\mathrm{~T}^{2}\right)$ such that $\mathrm{S}_{1}(\mathrm{H}) \subset \mathrm{H}, \mathrm{S}_{2}(\mathrm{H}) \subset \mathrm{H}$; and $\mathrm{S}_{1}, \mathrm{~S}_{2}$ are doubly commuting isometries on $\mathrm{H}$. Further, assume that $\langle., .\rangle_{\mathrm{H}}$ (the inner product on $\mathrm{H}$ ) satisfies the following property:

(P) For any functions $\mathrm{f}_{1}, \mathrm{f}_{2}, \mathrm{~g}_{1}, \mathrm{~g}_{2} \in \mathrm{H}$ such that $\left\langle\mathrm{f}_{1}, \mathrm{~g}_{1}\right\rangle_{\mathrm{H}}=\left\langle\mathrm{f}_{2}, \mathrm{~g}_{2}\right\rangle_{\mathrm{H}}$, then we have $\left\langle\mathrm{f}_{1}, \mathrm{~g}_{1}\right\rangle_{\mathrm{H}^{2}}=\left\langle\mathrm{f}_{2}, \mathrm{~g}_{2}\right\rangle_{\mathrm{H}^{2}}$.

Then there exists a unique inner function $b$ such that

$\mathrm{H}=\mathrm{bH}^{2}\left(\mathrm{~T}^{2}\right)$, and $\|\mathrm{bf}\|_{\mathrm{H}}=\|\mathrm{f}\|_{2}$ for every $\mathrm{f} \in \mathrm{H}^{2}\left(\mathrm{~T}^{2}\right)$.

Proof. Since the operators $\mathrm{S}_{1}$ and $\mathrm{S}_{2}$ are doubly commuting isometries on the Hilbert space $H$, so all the the conditions of Theorem 2.1 are satisfied and hence there exists $\mathrm{b} \in \mathrm{H}^{\infty}$ such that $\mathrm{H}=\mathrm{bH}^{2}\left(\mathrm{~T}^{2}\right)$, and $\|\mathrm{bf}\|_{\mathrm{H}}=\|\mathrm{f}\|_{2}$ for all $f \in \mathrm{H}^{2}\left(\mathrm{~T}^{2}\right)$. It remains to be shown that $\mathrm{b}$ is inner. The proof of Theorem 2.1, as given in [11], reveals that $\mathrm{H}$ has the Slocinski-Wold type decomposition:

$$
H=\sum_{m=0}^{\infty} \sum_{n=0}^{\infty} \oplus S_{1}^{m} S_{2}^{n}\left(L_{1} \cap L_{2}\right) .
$$

Here $\mathrm{L}_{\mathrm{k}}=\mathrm{H} \ominus \mathrm{S}_{\mathrm{k}}(\mathrm{H}), \mathrm{k}=1,2$. It turns out that $\mathrm{b}$ is a unit vector in $\mathrm{H}$ that spans $\mathrm{L}_{1} \cap \mathrm{L}_{2}$. From the above Wold type decomposition we see that $\left\langle\mathrm{b}, \mathrm{bz}^{\mathrm{m}} \mathrm{w}^{\mathrm{n}}\right\rangle_{\mathrm{H}}=0$ for all $(m, n) \neq(0,0)$. The arguments used to establish this fact are similar to the ones adopted in the proof of Lemma 2.2. This, in view of property $(\mathrm{P})$, implies that $\left\langle\mathrm{b}, \mathrm{bz} \mathrm{m}^{\mathrm{m}} \mathrm{w}^{\mathrm{n}}\right\rangle_{2}=0$ for all $(m, n) \neq(0,0)$. Thus the set $\left\{b z^{m} w^{n}: m \geq 0\right.$ and $n \geq$ $0\}$ is orthogonal in $\mathrm{H}^{2}\left(\mathrm{~T}^{2}\right)$. So the collection $\left\{\frac{\mathrm{b}}{\|\mathrm{b}\|_{2}} \mathrm{z}^{\mathrm{m}} \mathrm{w}^{\mathrm{n}}: \mathrm{m} \geq 0\right.$ and $\left.\mathrm{n} \geq 0\right\}$ is orthonormal in $\mathrm{H}^{2}\left(\mathrm{~T}^{2}\right)$. Hence by Lemma 2.2, the function $\frac{\mathrm{b}}{\|\mathrm{b}\|_{2}}$ is inner, and we have $\mathrm{H}=\mathrm{bH}^{2}\left(\mathrm{~T}^{2}\right)=\frac{\mathrm{b}}{\|\mathrm{b}\|_{2}} \mathrm{H}^{2}\left(\mathrm{~T}^{2}\right)$.

The above theorem clearly generalizes the two variable Beurling type result proved by Mandrekar [5]:

Corollary 3.2 ([5], Theorem 2). Let $M$ be a closed subspace of $\mathrm{H}^{2}\left(\mathrm{~T}^{2}\right)$ which is invariant under $\mathrm{S}_{1}$ and $\mathrm{S}_{2}$ and that $\mathrm{S}_{1}, \mathrm{~S}_{2}$ doubly commute on $\mathrm{M}$. Then there exists a unique inner function $b$ such that $\mathrm{M}=\mathrm{bH}^{2}\left(\mathrm{~T}^{2}\right)$.

Proof. Since the norm on $\mathrm{M}$ is the same as that on $\mathrm{H}^{2}$, so the property (P) stated in Theorem 3.1 is automatically satisfied and hence the result follows.

Theorem 3.3. Let $\mathrm{H}$ be a Hilbert space satisfying the conditions of Theorem 3.1, and $\mathrm{M}$ be a closed subspace of $\mathrm{H}$ which is invariant under $\mathrm{S}_{1}$ and $\mathrm{S}_{2}$. Then there exists a unique inner function $\phi$ such that $\mathrm{M}=\phi \mathrm{H}$.

Proof. By Theorem 3.1, there exists an inner function $b$ such that $\mathrm{H}=\mathrm{bH}^{2}\left(\mathrm{~T}^{2}\right)$. Since $\mathrm{M}$ is a closed subspace of $\mathrm{H}$, so $\mathrm{M}$ is also a Hilbert space satisfying the assumptions of Theorem 3.1. Thus there exists an inner function c such that $\mathrm{M}=\mathrm{cH}^{2}\left(\mathrm{~T}^{2}\right)$. Note that $\mathrm{c}=\mathrm{b} \phi$ for some $\phi \in \mathrm{H}^{2}\left(\mathrm{~T}^{2}\right)$. This implies that $|\phi|=1$ a.e. Further, $\mathrm{M}=\phi \mathrm{bH}^{2}\left(\mathrm{~T}^{2}\right)=\phi \mathrm{H}$.

\section{Acknowledgements}

The author thanks the Shiv Nadar University, Dadri, Uttar Pradesh (India), for support and facilities needed to complete the present work.

\section{References}

[1] A. Beurling, Two problems concerning linear transformations in Hilbert space, Acta Math. 81(1949), 239-255.

[2] P.L. Duren, Theory of Hp spaces, Academic Press, London-New York, 1970.

[3] K. Hoffman, Banach Spaces of Analytic Func- tions, Prentice Hall, Englewood Cliffs, New Jer- sey, 1962.

[4] P. Koosis, Introduction to Hp spaces, Cambridge University Press, Cambridge, 1998.

[5] V. Mandrakar, The validity of Beurling theorems in polydiscs, Proc. Amer. Math. Soc., 1988, 145- 148.

[6] D. A. Redett, Sub-Lebesgue Hilbert spaces on the unit circle, Bull. London Math. Soc. 37(2005), 793-800.

[7] N. Sahni and D. Singh, Invariant subspaces of cer- tain subhilbert space of H2, Proc. Japan Acad., 87, Ser. A (2011), 56-59. 
[8] D. Sarason, Shift-Invariant spaces from the Bran- gesian point of view, The Bieberbach Conjecture, Mathematical Surveys and Monographs, AMS, 21(1986), 53-166.

[9] H. S. Shapiro, Reproducing kernels and Beurling's theorem, Trans. Amer. Math. Soc. 110 (1964), 448-458.

[10] D. Singh and U.N. Singh, On a theorem of de Branges, Indian Journal of Math., 33(1991), $1-5$.
[11] D. Singh, Brangesian Spaces in the Polydisk, Proc. Amer. Math. Soc. 110(1990), 971-977.

[12] B. Yousefi and E. Hesameddini, Extension of the Beurling's Theorem, Proc. Japan Acad. 84 (2008), 167-169. 